\title{
Respuesta Cardiopulmonar a Cambios de Oxigenación en Recién Nacidos de Ovejas de Diferentes Altitudes
}

\author{
Emilio Herrera V. ${ }^{1,2,3}$, M.V., Ph.D., German Ebensperger D. ${ }^{1}$, B.Q., Ph.D., Raquel \\ Riquelme G. ${ }^{4}$, Q.F., Marcela Díaz N. ${ }^{1}$, Matr. Mg., Victor Reyes C. ${ }^{1}$,B.Q., Ph.D., \\ Claudia Torres-Farfan' ,B.Q., Ph.D., Aníbal Llanos M. ${ }^{1,3}$, M.C. \\ ${ }^{1}$ Laboratorio de Fisiología y Fisiopatología del Desarrollo, ${ }^{2}$ Laboratorio de Función y Reactividad \\ Vascular, Programa de Fisiopatología, Instituto de Ciencias Biomédicas (ICBM), Facultad de \\ Medicina, Universidad de Chile, Santiago, Chile. \\ ${ }^{3}$ International Center for Andean Studies (INCAS), Universidad de Chile, Santiago-Putre, \\ ${ }^{4}$ Departamento de Bioquímica y Biología Molecular, Facultad de Ciencias Químicas y \\ Farmacéuticas, Universidad de Chile, Santiago, Chile.
}

\begin{abstract}
Resumen
La exposición a la hipoxia crónica en el altiplano andino durante el período perinatal induce hipertensión pulmonar y cambios estructurales y funcionales en la circulación pulmonar y sistémica. El objetivo de este estudio fue analizar los efectos de la gestación y vida perinatal temprana a diferentes altitudes, en el sistema cardiovascular sistémico y pulmonar en recién nacidos de oveja. Corderos de tierras bajas (200m) y tierras altas (dos grupos: $3.600 \mathrm{~m}$ y $4.600 \mathrm{~m}$ ) fueron cateterizados bajo anestesia general y sometidos a episodios de hipoxia/hiperoxia. Gasometría arterial y venosa, frecuencia cardiaca, gasto cardiaco, presión arterial sistémica y pulmonar, y resistencia vascular sistémica y pulmonar fueron analizadas a diferentes niveles de oxigenación en corderos de 1-2 semanas de edad. En condiciones basales, los corderos de altura (3,600m y 4,600m) presentaron un menor peso, $\mathrm{PO}_{2}$ y $\mathrm{SaO}_{2}$. Ambos grupos de altura tuvieron un gasto cardiaco mayor y una menor resistencia vascular sistémica. La altura determinó un aumento significativo de la presión arterial pulmonar con cambios en la sensibilidad al oxígeno de la circulación pulmonar. Interesantemente, estos efectos fueron mayores en corderos de 3,600m que en el grupo de 4,600m. La hipoxia graduada demostró que la presión pulmonar media fue mayor en corderos de 3,600m a cualquier nivel de $\mathrm{PO}_{2}$. Existe una relación importante entre el peso del cordero y los efectos cardiovasculares sistémicos con la altura. Las respuestas pulmonares fueron importantes en ambos grupos de altura pero más marcadas en los corderos de 3,600m. Corderos provenientes de alturas extremas $(4,600 \mathrm{~m})$ han desarrollado una adaptación pulmonar particular, la cual les permite compensar la baja disponibilidad de oxígeno ambiental en el Altiplano Andino. La hipoxia crónica afecta la salud y producción del rebaño. Conocer los mecanismos involucrados en el desarrollo y establecimiento del sistema cardiovascular podría dar información para un mejor entendimiento de la fisiología y fisiopatología perinatal en tierras altas y en enfermedades que evolucionan con hipoxia crónica fetal y neonatal en tierras bajas. Esto podría dar claves para mejorar la producción animal local en poblaciones de tierras altas y entender la fisiopatología de la hipoxia crónica.
\end{abstract}

Palabras clave: Hypoxia, pulmonary hypertension, neonatal lamb, highlands

\section{Introducción}

La exposición a las grandes altitudes del altiplano andino induce importantes cambios en la circulación pulmonar y sistémica (Monge \& León 
Velarde, 1991). En respuesta a hipoxia hipobárica, la circulación pulmonar genera una importante vasoconstricción que resulta en hipertensión pulmonar y, a largo plazo, remodelamiento de los vasos pulmonares, reduciendo aún más el lumen vascular y aumentando la resistencia vascular pulmonar (Stenmark y cols., 2006; Gao y Raj, 2010; Herrera y cols, 2008a).

En teoría, los cambios en la resistencia pulmonar son adaptativos para generar un mejor acoplamiento entre la perfusión y la ventilación, generando un adecuado intercambio de gases alveolares (Prendergast \& Ruoss, 2000). Sin embargo, aumentos excesivos de la resistencia pulmonar mantenidos en el tiempo llevan a cambios estructurales de los vasos con aumento de la capa muscular media y fibrosis de la adventicia (Tucker y cols., 1975; Monge y León Velarde; 1991, Jeffery y Morrell., 2002; Herrera y cols, 2008a).

En casos extremos, la función pulmonar se ve comprometida con riesgo letal, asociado a hipertensión pulmonar con edema agudo en animales adultos, hipertensión pulmonar persistente del recién nacido, edema subagudo en la etapa juvenil y enfermedad de Brisket en ganado (Rhodes, 2005; Abman, 1999). Evidentemente estos cuadros respiratorios tienen un gran impacto en el bienestar, desarrollo y crecimiento del ganado en altura. De particular interés es la adaptación de la función pulmonar al parto y en el periodo neonatal, condición que determina la sobrevida y adecuado desarrollo del animal recién nacido. En este estudio investigamos la circulación pulmonar de recién nacidos de ovejas de 3 localidades distintas de la XV región de Arica-Parinacota: Lluta (200 msnm), Putre (3600 msnm) y Caquena (4600 $\mathrm{msnm})$. El objetivo de este trabajo fue evaluar si la gestación y nacimiento a diferentes alturas (niveles de hipoxia) determinan un deficiente desarrollo neonatal asociado a cambios cardiovasculares y cambios en la sensibilidad de la circulación pulmonar al oxígeno.

\section{Material y métodos}

\subsection{Animales}

Se estudiaron en este trabajo diez corderos, gestados y nacidos en el valle de Lluta (Liceo
Francisco Napolitano, 200 msnm, XV Región, Chile), siete corderos gestados y nacidos en Putre (Estación Experimental Putre del INCAS (acrónimo por Internacional Center for Andean Studies), Universidad de Chile, $3.600 \mathrm{msnm}$, XV Región, Chile) y 5 corderos gestados y nacidos en Caquena (4.600 msnm, XV Región, Chile). Todos los corderos fueron alimentados por sus madres y ellas permanecieron con alimento (alfalfa) y agua de beber ad libitum.

\subsection{Preparación quirúrgica}

Entre 4-7 días de edad, los corderos fueron premedicados con atropina $\left(0.04 \mathrm{mg} \mathrm{kg}^{-1}\right.$, i.m., Atropina Sulfato, Laboratorio Chile, Santiago, Chile), y anestesiados con una asociación de ketamina (5-10 mg.kg; Ketostop, Drag PharmaInvectec, Santiago, Chile) y xilazina (0.5 mg.kg) I.M. Catéteres de polivinilo (1.0 mm i.d.) fueron insertados en aorta abdominal y vena cava posterior vía arteria y vena femoral, respectivamente. Estos catéteres fueron llenados de $\mathrm{NaCl} 0.9 \%$ heparinizado (100 UI) y su extremo distal cerrado con un sello de cobre. Luego fueron canalizados subcutáneamente y exteriorizados a nivel del flanco donde se ubicaron en un bolsillo adherido a la piel. Posteriormente, un catéter cardiopulmonar Swan-Ganz (Edwards Swan-Ganz 5 French, Baxter Healthcare Corporation, Irvine, CA, USA) fue implantado en arteria pulmonar vía yugular externa. Este catéter se llenó con $\mathrm{NaCl}$ $0.9 \%$ heparinizado (500 UI) y se mantuvo en una bufanda con bolsillo instalado en el cuello del neonato.

Este procedimiento quirúrgico tiene una duración de aproximadamente $30 \mathrm{~min}$ y en general 1-2 $\mathrm{h}$ después del procedimiento, el cordero se encontraba recuperado de la anestesia. El cuidado postquirúrgico se basó en dosis profilácticas de antibióticos (Ampicilina $10 \mathrm{mg} \cdot \mathrm{kg}^{-1}$ i.v, Laboratorio Best-Pharma, Santiago, Chile; Gentamicina $4 \mathrm{mg} \cdot \mathrm{kg}^{-1}$ i.v., Laboratorio Biosano S.A., Santiago, Chile), administrados cada $24 \mathrm{~h}$ mientras los animales permanecieron instrumentados. Se esperaron al menos 3 días postoperatorios para comenzar los experimentos. Los corderos siempre se mantuvieron en corrales con sus madres.

\subsection{Protocolo experimental}


Los experimentos de los corderos recién nacidos de Lluta se realizaron en el Liceo Francisco Napolitano y los corderos nacidos en Putre y Caquena en la Estación Experimental Putre, Universidad de Chile, a 3600 m de altitud. Estos fueron basados en modificaciones de la fracción inspiratoria de oxígeno $\left(\mathrm{FiO}_{2}\right)$ durante 3-5 min. Para poder alterar la $\mathrm{FiO}_{2}$, una máscara (tipo casco) de plástico transparente fue adaptada en la cabeza del neonato a través de la cual se administró una mezcla de gases $\left(\mathrm{O}_{2}\right.$ y $\mathrm{CO}_{2}$ en $\mathrm{N}_{2}$ ca $10 \mathrm{~L} \mathrm{~min}^{-1}$ ) para generar diferentes niveles de hipoxia isocápnica e hiperoxia. Durante todo el período experimental se registró continuamente la presión arterial pulmonar (PAP) y la presión arterial sistémica (PAS), las cuales fueron almacenadas mediante un sistema de adquisición de datos conectado a un PC (Powerlab/8SP System and Chart v4.1.2 Software; ADInstruments, New South Wales, Australia). Además, después de la estabilización (3-5 min) de las presiones se determinó el gasto cardiaco (GC) por el método de termodilución como un promedio de al menos tres determinaciones con una inyección de $3 \mathrm{ml} \mathrm{NaCl}$ a $0^{\circ} \mathrm{C}$ (Baxter COM-2 cardiac output computer, Irvine, CA, USA).

En cada uno de las diferentes escalas de $\mathrm{FiO}_{2}$, se determinó $\mathrm{pH}, \mathrm{PO}_{2}, \mathrm{PCO}_{2}$ (ABL 555, Blood gas Monitor, Radiometer, Copenhagen, Dinamarca; corregido a $\left.39^{\circ} \mathrm{C}\right)$, concentración de hemoglobina ([Hb]), porcentaje de saturación de hemoglobina $\left(\mathrm{SaO}_{2}\right)$, porcentaje de hemoglobina unida a monóxido de carbono (COHb), contenido de oxígeno arterial y venoso corregido por afinidad de hemoglobina (OSM3 Hemoximeter, Radiometer, Copenhagen, Dinamarca).

La resistencia vascular sistémica (RVS) y pulmonar (RVP) fueron calculadas utilizando las siguientes formulas:

RVS $=\frac{\text { mPAS-presión aurícula derecha }(\mathrm{mmHg})}{\text { Gasto cardiaco }\left(\mathrm{ml} \bullet \mathrm{min}^{-1} \bullet \mathrm{kg}^{-1}\right)}$

$\mathrm{RVP}=\underline{\text { mPAP}}$ - presión de enclavamiento $(\mathrm{mmHg})$ Gasto cardiaco $\left(\mathrm{ml} \cdot \mathrm{min}^{-1} \cdot \mathrm{kg}^{-1}\right)$

Todos los cuidados y procedimientos fueron llevados a cabo siguiendo los principios internacionales descritos en "The Guiding Principles for Research Involving Animals and Human Beings of the American Physiological Society" y fueron previamente aprobados por el Comité de Ética de la Facultad de Medicina de la Universidad de Chile (Protocolo CBA Nº 097, FMUCH).

\subsection{Análisis de datos}

Todos los valores fueron expresados como medias \pm E.S.M. Los resultados basales fueron analizados utilizando un análisis de varianza (ANOVA), y las diferencias fueron evaluadas por el post-hoc Newman-Keuls test. El análisis de las correlaciones de $\mathrm{PO}_{2}$ y PAP, GC o RVP fue realizado con un ajuste a regresión lineal y un test de correlación de Pearson. La pendiente e intercepto de las curvas se analizaron con un ttest. Las diferencias fueron consideradas significativas cuando $p<0.05$ [Glantz \& Slinker, 2001].

\section{Resultados}

Los recién nacidos estudiados presentaron una clara disminución del peso asociada a la altura, a mayor altura menor peso a edades similares (Tabla 1).

Tabla 1. Peso y edad el día de estudio de recién nacidos de oveja de diferentes altitudes.

\begin{tabular}{llll}
\hline & $200 \mathrm{~m}$ & $3600 \mathrm{~m}$ & $4600 \mathrm{~m}$ \\
\hline Peso, kg & $7.0 \pm 0.4$ & $5.6 \pm 0.4^{*}$ & $3.8 \pm 0.4^{*} \dagger$ \\
Edad, & $7-12$ & $7-15$ & $7-14$ \\
días & $(10.5)$ & $(11.8)$ & $(11.8)$ \\
\hline
\end{tabular}

Características de los recién nacidos el día de estudio. Valores son expresados como promedios \pm E.S.M. Diferencias significativas $(\mathrm{P}<0.05)$ : * vs 200m, † vs 3600m.

\subsection{Gasometría arterial}

Los corderos de tierras bajas presentaron una gasometría normal para la edad y altura a la cual 
fueron estudiados (Tabla 2) (Herrera y cols, 2007, 2008a).

En gran contraste, tanto los corderos de $3600 \mathrm{~m}$ como los de $4600 \mathrm{~m}$ presentaron un aumento del $\mathrm{pH}$ y una disminución de la presión parcial de oxígeno arterial $\left(\mathrm{PO}_{2}\right)$, la saturación de la hemoglobina por oxígeno $\left(\mathrm{SaO}_{2}\right)$ y la carboxihemoglobina (COHb). Interesantemente, la presión parcial de dióxido de carbono arterial
$\left(\mathrm{PCO}_{2}\right)$ cayó sólo en los corderos de 3600m, mientras que el contenido de oxígeno en sangre (cont $\mathrm{O}_{2}$ ) descendió en ambos corderos de altura pero con mayor magnitud en los corderos de $3600 \mathrm{~m}$ en comparación con los animales de tierras bajas. Esto se vio asociado a un aumento en la concentración de hemoglobina sanguínea $(\mathrm{Hb})$, sólo observada en los corderos de 4600m (Tabla 2).

Tabla 2. Gases arteriales basales de recién nacidos de oveja de diferentes altitudes.

\begin{tabular}{lccc}
\hline & $200 \mathrm{~m}$ & $3600 \mathrm{~m}$ & $4600 \mathrm{~m}$ \\
\hline $\mathrm{pH}$ & $7.41 \pm 0.01$ & $7.46 \pm 0.01^{*}$ & $7.47 \pm 0.01^{*}$ \\
$\mathrm{PCO}_{2}, \mathrm{mmHg}$ & $36.8 \pm 1.1$ & $31.6 \pm 1.8^{*}$ & $34.0 \pm 0.8$ \\
$\mathrm{PO}_{2}, \mathrm{mmHg}$ & $79.0 \pm 2.3$ & $41.6 \pm 2.8^{*}$ & $42.7 \pm 1.3^{*}$ \\
$\mathrm{Hb}, \mathrm{g.dL}$ & $11.4 \pm 0.8$ & $12.1 \pm 0.7^{-1}$ \\
$\mathrm{SaO}_{2}, \%$ & $10.9 \pm 0.5$ & $67.4 \pm 2.9^{*}$ & $71.5 \pm 3.2^{*}$ \\
$\mathrm{Cont}^{\mathrm{O}} \mathrm{O}_{2}, \mathrm{~mL} . \mathrm{dL}^{-1}$ & $94.4 \pm 0.7$ & $10.6 \pm 0.8^{*}$ & $12.0 \pm 0.5^{*} \dagger$ \\
$\mathrm{COHb}, \%$ & $13.9 \pm 0.6$ & $0.15 \pm 0.07^{*}$ & $0.00 \pm 0.00^{*}$ \\
\hline
\end{tabular}

Gases arteriales basales de los recién nacidos. Valores son expresados como promedios \pm E.S.M. Diferencias significativas $(\mathrm{P}<0.05)$ : * vs $200 \mathrm{~m}$, † vs $3600 \mathrm{~m}$.

\subsection{Variables cardiovasculares}

Los corderos de tierras bajas presentaron basalmente variables cardiovasculares consistentes con la edad y con un animal sano (Fig. 1, 2 y 3) (Herrera y cols, 2007, 2008). A pesar de que no observamos diferencias basales de frecuencia cardiaca (FC) entre los diferentes grupos experimentales, el gasto cardiaco (GC) aumentó significativamente con la altura (Fig. 1). Esto se asoció a una caída de la presión arterial sistémica media (mPAS) sólo en los corderos de $4600 \mathrm{~m}$. Sin embargo, ambos corderos de altura presentaron una menor resistencia vascular sistémica (RVS) comparada con la RVS de los corderos de tierras bajas (Fig. 2).

Los corderos de altura presentaron un similar aumento de la presión arterial pulmonar (mPAP) basal comparado con los recién nacidos de tierras bajas. Sin embargo, los recién nacidos de 3600m presentaron una mayor mPAP basal que los corderos de $4600 \mathrm{~m}$. Lo anterior se vio también reflejado en la resistencia vascular pulmonar (RVP) que aumentó en ambos grupos de altura 
pero con una mayor magnitud en los corderos de 3600m (Fig. 3).
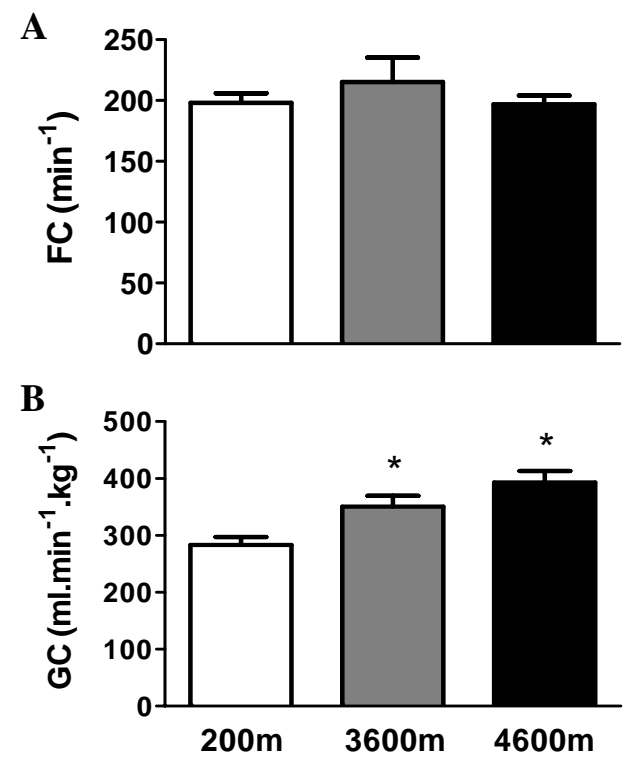

Figura 1. Función cardiaca basal de recién nacidos de oveja de diferentes altitudes

Variables cardiacas basales de los recién nacidos. A. Frecuencia cardiaca (FC), B. Gasto cardiaco (GC). Valores son expresados como promedios \pm E.S.M. Diferencias significativas $(\mathrm{P}<0.05)$ * * vs $200 \mathrm{~m}$.

A
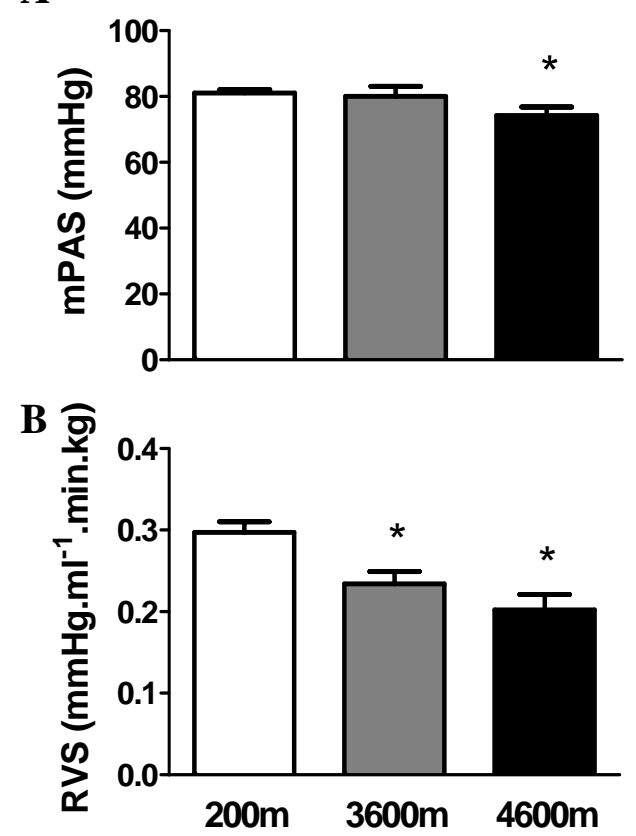

Figura 2. Función cardiovascular basal de recién nacidos de oveja de diferentes altitudes.
Variables cardiovasculares sistémicas basales de los recién nacidos. A. Presión arterial sistémica media (mPAS), B. Resistencia vascular sistémica (RVS). Valores son expresados como promedios \pm E.S.M. Diferencias significativas $(\mathrm{P}<0.05)$ : * vs $200 \mathrm{~m}$.

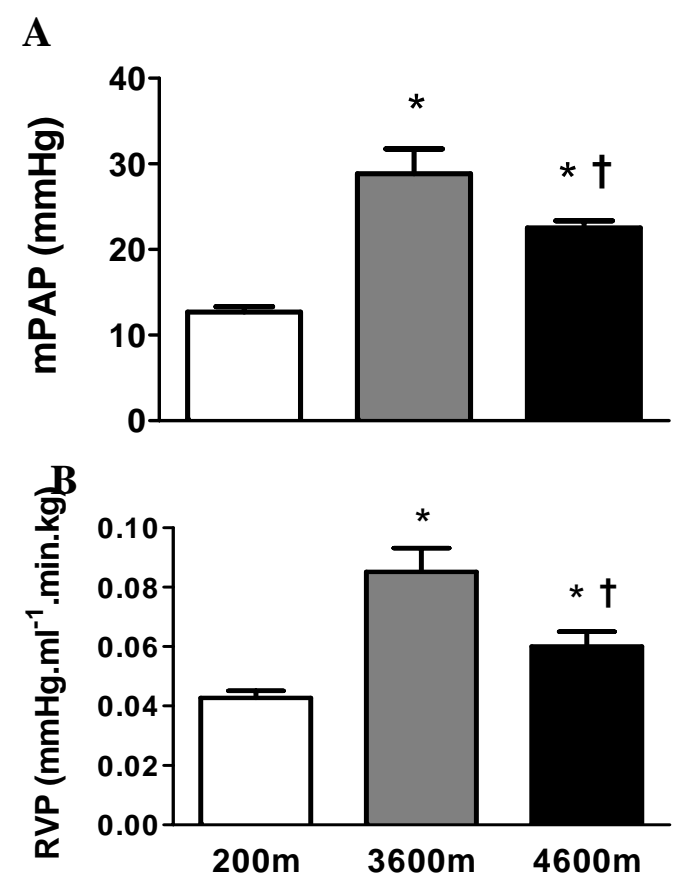

Figura 3. Función cardiopulmonar basal de recién nacidos de oveja de diferentes altitudes.

Variables cardiopulmonares basales de los recién nacidos. A. Presión arterial pulmonar media (mPAP), B. Resistencia vascular pulmonar (RVP). Valores son expresados como promedios \pm E.S.M. Diferencias significativas $(\mathrm{P}<0.05)$ * vs $200 \mathrm{~m}$, $\dagger$ vs $3600 \mathrm{~m}$.

\subsection{Sensibilidad al oxígeno}

Para el análisis de sensibilidad del sistema cardiovascular pulmonar a cambios de oxígeno, se graficaron curvas de correlación de mPAP, GC y RVP vs. $\mathrm{PO}_{2}$ de la arteria pulmonar. En todos los puntos de la curva, a una determinada $\mathrm{PO}_{2}$, los corderos de $3600 \mathrm{~m}$ presentaron valores de mPAP significativamente superiores en comparación con los corderos de $200 \mathrm{~m}$ (intercepto $35.7 \pm 1.4$ vs. $37.7 \pm 1.4 ; \quad \mathrm{p}<0.05, \quad 3600 \mathrm{~m}$ vs. $200 \mathrm{~m}$ respectivamente).

En contraste, los corderos de $4600 \mathrm{~m}$ presentaron una curva con un origen (a bajas $\mathrm{PO}_{2}$ ) similar a los animales de $3600 \mathrm{~m}$ pero con un extremo (a 
altas $\mathrm{PO}_{2}$ ) similar a los corderos de tierras bajas (pendiente $-0.62 \pm 0.07$ vs. $-0.53 \pm 0.04 ; \mathrm{p}<0.05$, $4600 \mathrm{~m}$ vs. $200 \mathrm{~m}$, respectivamente) (Fig. $4 \mathrm{~A}$ ). En todos los grados de oxigenación, los corderos de $4600 \mathrm{~m}$ presentaron un mayor GC (intercepto 537 \pm 27 vs. $433 \pm 18$ vs. $413 \pm 18 ; \mathrm{p}<0.05,4600 \mathrm{~m}$ vs. $3600 \mathrm{~m}$ y $200 \mathrm{~m}$ ) (Fig. 4B) y una RVP comparable a los corderos de 200m (Fig. 4C). En contraste en todos los puntos de la curva, los corderos de $3600 \mathrm{~m}$ tienen una RVP más alta que los corderos de $200 \mathrm{~m}$ y $4600 \mathrm{~m}$ (intercepto $0.094 \pm$ 0.006 vs. $0.085 \pm 0.004$ vs. $0.078 \pm 0.007 ; \mathrm{p}<$ $0.05,3600 \mathrm{~m}$ vs. $200 \mathrm{~m}$ y $4600 \mathrm{~m}$ ) (Fig. 4C). El análisis de todas las curvas dio una correlación significativa $(\mathrm{P}<0.0001)$ y las funciones fueron las siguientes:

PAP vs $\mathrm{PO}_{2} .200 \mathrm{~m}, \mathrm{y}=-0.5350 \mathrm{x}+32.72, \mathrm{R}^{2}=$ $0.578(\mathrm{r}=0.760) ; 3600 \mathrm{~m}, \mathrm{y}=-0.4300 \mathrm{x}+35.69, \mathrm{R}^{2}$ $=0.402(\mathrm{r}=0.634) ; 4600 \mathrm{~m}, \mathrm{y}=-0.6155 \mathrm{x}+38.49$, $\mathrm{R}^{2}=0.462(\mathrm{r}=0.680)$.

GC vs $\mathrm{PO}_{2} .200 \mathrm{~m}, \mathrm{y}=-3.384 \mathrm{x}+412.9, \mathrm{R}^{2}=$ $0.237(\mathrm{r}=0.487) ; 3600 \mathrm{~m}, \mathrm{y}=-3.488 \mathrm{x}+433.7, \mathrm{R}^{2}$ $=0.196(\mathrm{r}=0.443) ; 4600 \mathrm{~m}, \mathrm{y}=-4.309 \mathrm{x}+537.4$, $\mathrm{R}^{2}=0.203(\mathrm{r}=0.451)$.

RVP vs $\mathrm{PO}_{2} .200 \mathrm{~m}, \mathrm{y}=-0.0011 \mathrm{x}+0.085, \mathrm{R}^{2}=$ $0.376(\mathrm{r}=0.613) ; 3600 \mathrm{~m}, \mathrm{y}=-0.0007 \mathrm{x}+0.094, \mathrm{R}^{2}$ $=0.092(\mathrm{r}=0.303) ; 4600 \mathrm{~m}, \mathrm{y}=-0.0009 \mathrm{x}+0.078$, $\mathrm{R}^{2}=0.149(\mathrm{r}=0.386)$.

Figura 4. Relación de las variables cardiopulmonares con diferentes niveles de oxigenación en recién nacidos de oveja de diferentes altitudes.

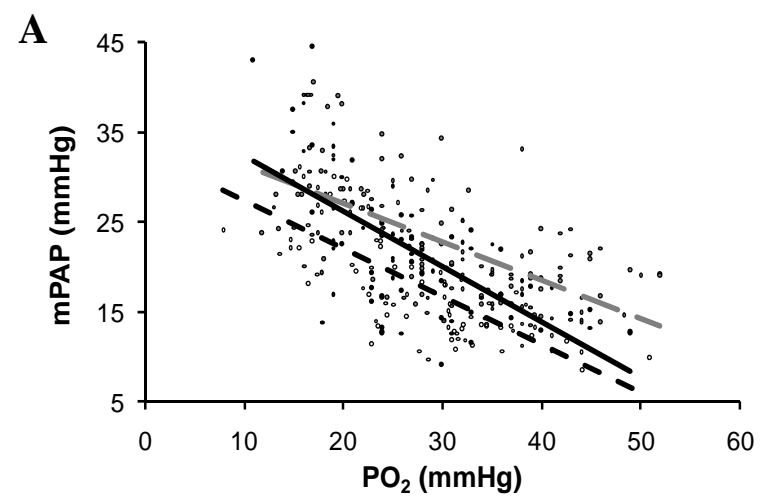

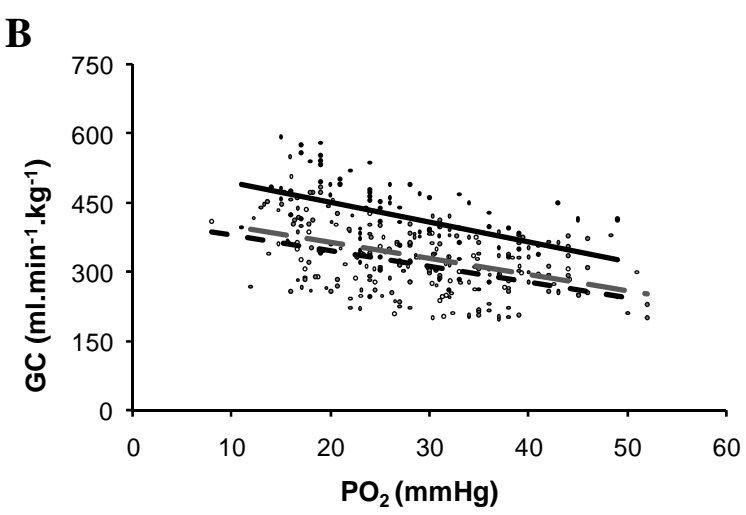

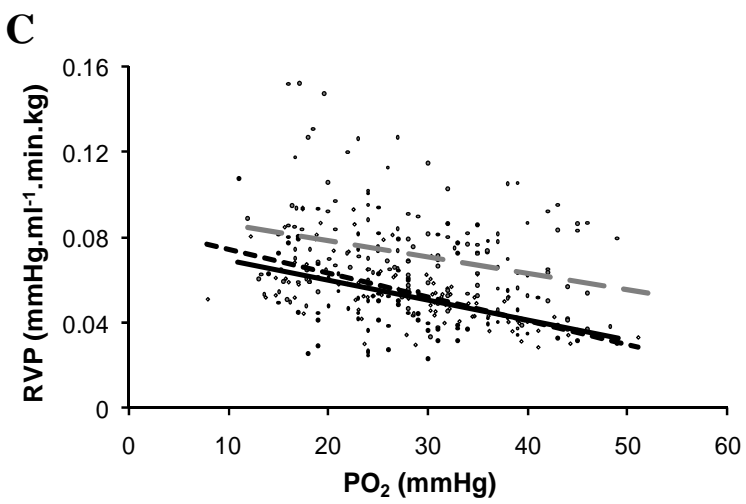

Relación de variables cardiopulmonares con $\mathrm{PO}_{2}$ arterial de los recién nacidos. A. Presión arterial pulmonar media (mPAP) vs $\mathrm{PO}_{2}$, B. Gasto cardiaco (GC) vs $\mathrm{PO}_{2}$, Resistencia vascular pulmonar (RVP) vs $\mathrm{PO}_{2}$. Valores son expresados como promedios \pm E.S.M. 200m: guión corto negro; 3600m: guión largo gris; 4600m: línea continua negra (ver significaciones en el texto).

\section{DISCUSION}

Los resultados de este trabajo muestran que los corderos que han sido gestados y nacidos en altura presentan alteraciones en el peso al nacimiento, el desarrollo postnatal y la función cardiovascular. Más aun, por primera vez se comprueba que las diferencias cardiovasculares sistémicas se hacen más intensas a medida que aumenta la altura a la cual habitan estos animales. En contraste, el sistema circulatorio pulmonar parece tener una respuesta adaptativa ya que, a pesar de presentar hipertensión pulmonar en ambas alturas altiplánicas, los cambios son menos acentuados a $4600 \mathrm{~m}$. 
Este estudio comprueba que mientras mayor es la altura a la cual transcurre la gestación, y por lo tanto mayor nivel de hipoxia hipobárica, menor es el peso al nacimiento del cordero. Estudios previos han demostrado la restricción de crecimiento intrauterino debido a hipoxia crónica (Parraguez y cols., 2005; Herrera y cols., 2007; Julian y cols., 2007; Keyes y cols., 2003; Giussani y cols., 2001), sin embargo este es el primer estudio que compara esta característica en rebaños similares de ovejas. Esto es de suma importancia ya que un menor peso al nacimiento determina una menor sobrevida y productividad del rebaño (Fahey y cols., 2005; Gardner y cols., 2007; Nash y cols., 1996; Gama y cols., 1991). Con respecto a este punto, un estudio realizado en animales de altura encontró una fuerte asociación entre el peso al nacimiento y la mortalidad neonatal (Bekele y cols., 1992).

Los corderos de altura presentan una alcalemia sanguínea, característica de una hiperventilación en altura. Importantemente la $\mathrm{PCO}_{2}$ disminuyó significativamente sólo en los corderos de $3600 \mathrm{~m}$, mientras que los de $4600 \mathrm{~m}$ no presentan hipocapnia comparado con los de tierras bajas. Como la $\mathrm{PCO}_{2}$ es un índice indirecto de la ventilación alveolar, los corderos de $4600 \mathrm{~m}$ pueden estar presentando una menor respuesta ventilatoria a la hipoxia crónica (blunted response) descrita en las poblaciones de grandes altitudes (León-Velarde y Richalet, 2006). A pesar de esto, los corderos de $4600 \mathrm{~m}$ son capaces de mantener la $\mathrm{SaO}_{2}$ en valores similares a los encontrados en corderos de $3600 \mathrm{~m}$, e incluso presentan un contenido sanguíneo de $\mathrm{O}_{2}$ mayor que sus pares de $3600 \mathrm{~m}$. Esto se debe a un discreto desplazamiento a la izquierda de la curva de disociación de la hemoglobina por oxígeno y al incremento en la concentración de hemoglobina en los corderos de $4600 \mathrm{~m}$. Este aumento de hemoglobina se debe a una eritrocitosis gatillada por hipoxia crónica, lo que favorece un mayor y mejor transporte gaseoso en estos corderos (Ou y cols., 1992). El monóxido de carbono es un gas que puede ser sintetizado a nivel pulmonar y que facilita la vasodilatación de esta circulación. Este gas tiene una afinidad a la hemoglobina mucho mayor que el oxígeno, lo que genera la carboxihemoglobina (Nachar y cols., 2001). La disminución de $\mathrm{COHb}$ en los corderos de altura indica que este gas vasodilatador esta disminuido, lo que favorece a la hipertensión pulmonar que estos animales presentan (Herrera y cols., 2008b).
A pesar de que los 3 grupos de corderos presentaron basalmente la misma frecuencia cardiaca, los corderos de altura presentaron un aumento proporcional a la altura en el gasto cardiaco, por lo que el volumen expulsivo de estos animales está aumentado. De esta manera, en hipoxia crónica, estos recién nacidos son capaces de aumentar el transporte sanguíneo, entre otras adaptaciones, a través de un aumento en el flujo sanguíneo por aumento del volumen expulsivo cardiaco. La presión sanguínea sistémica tuvo una disminución en los corderos de $4600 \mathrm{~m}$, presentando una caída masiva de la resistencia vascular sistémica, probablemente debido a factores vasodilatadores periféricos como óxido nítrico, el factor hiperpolarizante endotelial y/o prostaciclina (Spieker y cols., 2006; Cohen y Vanhoutte, 1995). La caída de la resistencia vascular sistémica, pero en menor grado, también fue observada en los corderos de $3600 \mathrm{~m}$, sin cambios en la presión sistémica, debido probablemente a una menor activación por hipoxia de los factores vasodilatadores periféricos antes descritos.

Debido a que la función pulmonar es extremadamente sensible a cambios de oxígeno, en la circulación pulmonar se vieron grandes cambios. La presión y resistencia vascular pulmonar se duplicaron en los corderos de tierras altas comparados con los de $200 \mathrm{~m}$. De interés, los cambios en la circulación pulmonar fueron mayores en los corderos de $3600 \mathrm{~m}$ que en los de $4600 \mathrm{~m}$. Esto puede ser explicado debido a que los recién nacidos de $4600 \mathrm{~m}$ hayan adquirido una adaptación especial por una larga estadía en las grandes altitudes de sus ancestros, que les permita regular mejor la mPAP y RVP en niveles fisiológicos para una adecuada función pulmonar. En la literatura se han descrito presiones pulmonares de hasta $70 \mathrm{mmHg}$ en recién nacidos humanos (Gamboa y Marticorena, 1971), por lo que son importantes los mecanismos adaptativos que estos corderos puedan desarrollar para el entendimiento de esta condición en otras especies de tierras bajas.

Se ha descrito que el oxido nítrico, un potente vasodilatador pulmonar es regulado por oxígeno. A saber, la oxido nítrico sintasa endotelial, enzima encargada de generar óxido nítrico en el endotelio pulmonar, aumenta con hipoxia crónica (Herrera, 2007; Herrera y cols., 2008b) en corderos de altura. Además, el estrés tangencial generado por 
un aumento de flujo sanguíneo (gasto cardiaco) también incrementa la actividad de esta enzima (Ghanayem y Gordon, 2001). Por lo tanto, una disminución de oxígeno ambiental, determinará ambos, una mayor expresión e indirectamente una activación de esta enzima, generando entonces mayor vasodilatación. Este aumento en la capacidad vasodilatadora pulmonar mediada por NO compensa parcialmente la hipertensión pulmonar, manteniéndose la resistencia vascular pulmonar elevada (Herrera, 2007). Por lo tanto, uno de los posibles mecanismos mediante los cuales los corderos de $4600 \mathrm{~m}$ regulen sus PAP es a través de un aumento de la expresión o actividad de NOS (Herrera y cols., 2008b), así como se ha demostrado en poblaciones de altura en el Tibet (Erzurum y cols., 2007). Es posible que esto se vea asociado a una menor remodelamiento de las arterias pulmonares de los corderos de 4600m comparado con los corderos de $3600 \mathrm{~m}$, donde las arterias tendrán una mayor distensibilidad y por consecuencia una mayor capacidad vasodilatadora en los animales de 4600m (Herrera y cols., 2008; Stenmark y cols., 2006).

Sin duda, lo más destacable de este estudio es el hallazgo de que la circulación pulmonar de recién nacidos de diferentes alturas presenta diferente sensibilidad al oxígeno. Aunque no están del todo claros los mecanismos que determinan la respuesta a hipoxia en este territorio, probablemente un proceso de remodelamiento pulmonar, donde las arterias están más muscularizadas, podría determinar una mayor presión en oxigenaciones similares (Herrera y cols., 2007; Stenmark y cols., 2006). Más aún, los corderos de $4600 \mathrm{~m}$ presentan una menor sensibilidad a oxígeno que los de $3600 \mathrm{~m}$, por lo que podrían tener sistemas compensatorios a la hipertensión o un remodelamiento en menor grado. En futuros estudios abordaremos esta pregunta mediante estudios histomorfométricos de las arterias pulmonares. Otra opción es que los recién nacidos de altura tengan un grado de estrés oxidativo diferente, condición que en parte también regula el tono vascular pulmonar (Chen y Keany, 2004; Resta y cols., 2010; Chi y cols., 2010; Evans y cols., 2011). Esto sugiere que la formación de especies reactivas de oxígeno (ej. $\mathrm{O}_{2-}, \mathrm{H}_{2} \mathrm{O}_{2}$ ) son determinantes en la generación de un estado vasocontraído. De hecho, Fike y cols. (2008) demostraron que lechones recién nacidos sometidos a hipoxia crónica presentaban un mayor estrés oxidativo asociado a una mayor expresión de la enzima NADPH oxidasa activa, una de las grandes generadoras de especies reactivas de oxígeno. Siguiendo la misma línea, una disminución de la capacidad antioxidante celular también podría inducir efectos similares (Dennis y cols., 2009). Las disponibilidad de $\mathrm{Ca}^{2+}$ intracelular favorece la vasoconstricción, lo que se ha demostrado que aumenta en condiciones de hipoxia, debido a una mayor expresión de canales tipo L que favorecen la depolarización de la membrana muscular lisa y por lo tanto, la contracción de estas células (Weir y cols., 2010).

Todos estos mecanismos pueden estar participando en los recién nacidos estudiados. Futuros estudios en nuestro laboratorio se enfocarán en resolver algunas de estas preguntas. Hemos demostrado que los corderos recién nacidos de altura se ven fuertemente afectados por la hipoxia hipobárica del altiplano. A pesar de que desarrollan una aclimatación o adaptación a la hipoxia, estos corderos tienen una marcada disminución del peso al nacimiento y desarrollo. Esto se vio asociado a importantes cambios cardiovasculares y pulmonares. Poder descubrir los mecanismos fisiológicos que determinan la respuesta a la hipoxia en el altiplano provee de importante información para lograr prevenir o tratar a los corderos. Esto permitiría que la producción ganadera local no se vea afectada por mermas debido a una alta mortalidad y morbilidad postnatal, ocurridas en condiciones de altura.

\section{Agradecimientos}

Este manuscrito está dedicado especialmente a Raquel Antonieta Riquelme González (QEPD), colega y amiga que dedicó con excelencia gran parte de su vida a estudiar los efectos de la hipoxia durante el período fetal y neonatal.

Agradecemos la excelente ayuda técnica de los Sres. Carlos Brito y Gavino Llusco. Estamos muy agradecidos por la colaboración y participación del Liceo Agrícola Francisco Napolitano, Valle de Lluta, Arica, en especial a los Sres. Carlos López, Jaime Figueroa, José López y Patricio Alvarez.

Este trabajo fue financiado por los proyectos FONDECYT 1050479, 1080663 y 1110595. 


\section{Referencias}

1. Abman S. H. 1999. Abnormal Vasoreactivity in the Pathophysiology of Persistent Pulmonary Hypertension of the Newborn. Pediatr Rev. 20: e103109.

2. Bekele T, Kasali OB, Woldeab T. 1992. Causes of lamb morbidity and mortality in the Ethiopian highlands. Vet Res Commun. 16(6):415-24.

3. Chen K, Keaney J. 2004. Reactive oxygen species-mediated signal transduction in the endothelium. Endothelium 11:109-121.

4. Chi AY, Waypa GB, Mungai PT, Schumacker PT. 2010. Prolonged hypoxia increases ROS signaling and RhoA activation in pulmonary artery smooth muscle and endothelial cells. Antioxid Redox Signal. 12(5):603-10.

5. Cohen RA, Vanhoutte PM. 1995. Endothelium-dependent hyperpolarization. Beyond nitric oxide and cyclic GMP. Circulation. 92(11):3337-49.

6. Dennis KE, Aschner JL, Milatovic D, Schmidt JW, Aschner M, Kaplowitz MR, Zhang Y, Fike CD. 2009. NADPH oxidases and reactive oxygen species at different stages of chronic hypoxiainduced pulmonary hypertension in newborn piglets. Am J Physiol Lung Cell Mol Physiol. 297(4):L596-607.

7. Erzurum SC, Ghosh S, Janocha AJ, Xu W, Bauer S, Bryan NS, Tejero J, Hemann C, Hille R, Stuehr DJ, Feelisch M, Beall CM. 2007. Higher blood flow and circulating NO products offset highaltitude hypoxia among Tibetans. Proc Natl Acad Sci U S A. 104(45):17593-8.

8. Evans AM, Hardie DG, Peers C, Mahmoud A. 2011. Hypoxic pulmonary vasoconstriction: mechanisms of oxygen-sensing. Curr Opin Anaesthesiol. 24(1):13-20.
9. Fahey AJ, Brameld JM, Parr T, Buttery PJ. 2005. The effect of maternal undernutrition before muscle differentiation on the muscle fiber development of the newborn lamb. J Anim Sci. 83(11):2564-71.

10. Fike CD, Slaughter JC, Kaplowitz MR, Zhang Y, Aschner JL. 2008. Reactive oxygen species from NADPH oxidase contribute to altered pulmonary vascular responses in piglets with chronic hypoxia-induced pulmonary hypertension. Am J Physiol Lung Cell Mol Physiol. 295(5):L881-8.

11. Gama LT, Dickerson GE, Young LD, Leymaster KA. 1991. Effects of breed, heterosis, age of dam, litter size, and birth weight on lamb mortality. J Anim Sci. 69(7):2727-43.

12. Gamboa R, Marticorena E. 1971. Pulmonary arterial pressure in newborn infants in high altitude. Arch Inst Biol Andina. 4(2):55-66.

13. Gardner DS, Buttery PJ, Daniel Z, Symonds ME. 2007. Factors affecting birth weight in sheep: maternal environment. Reproduction. 133(1):297307.

14. Gao Y, Raj JU. 2010. Regulation of the pulmonary circulation in the fetus and newborn. Physiol Rev. 90(4):1291-335.

15. Ghanayem NS, Gordon JB. 2001. Modulation of pulmonary vasomotor tone in the fetus and neonate. Respir Res. 2: 139-144.

16. Giussani DA, Phillips PS, Anstee S, Barker DJ. 2001. Effects of altitude versus economic status on birth weight and body shape at birth. Pediatr Res. 49(4):490-4.

17. Glantz SA \& Slinker BK. 2001. Primer of Applied Regression \& Analysis of Variance, 2nd ed., Mc Graw-Hill INC Medical Publishing Division, New York USA, pp. 418-507. 
18. Herrera EA, Ebensperger G, Krause BJ, Riquelme RA, Reyes RV, Capetillo M, González S, Parer JT, Llanos AJ. 2008(a). Sildenafil reverses hypoxic pulmonary hypertension in highland and lowland newborn sheep. Pediatr Res. 63(2):169-75.

19. Herrera EA, Reyes RV, Giussani DA, Riquelme RA, Sanhueza EM, Ebensperger G, Casanello P, Méndez N, Ebensperger R, Sepúlveda-Kattan E, Pulgar VM, Cabello G, Blanco CE, Hanson MA, Parer JT, Llanos AJ. 2008(b). Carbon monoxide: a novel pulmonary artery vasodilator in neonatal llamas of the Andean altiplano. Cardiovasc Res. 77(1):197-201.

20. Herrera EA, Pulgar VM, Riquelme RA, Sanhueza EM, Reyes RV, Ebensperger G, Parer JT, Valdéz EA, Giussani DA, Blanco CE, Hanson MA, Llanos AJ. 2007. High-altitude chronic hypoxia during gestation and after birth modifies cardiovascular responses in newborn sheep. Am J Physiol Regul Integr Comp Physiol. 292(6):R2234-40.

21. Herrera EA. Regulación de la circulación pulmonar por el sistema óxido nítrico sintasa-óxido nítrico en el recién nacido de oveja crónicamente hipóxico Tesis de doctorado. Facultad de Medicina, Universidad de Chile. 2007.

22. Jeffery TK, Morrell NW. 2002. Molecular and cellular basis of pulmonary vascular remodeling in pulmonary hypertension. Prog Cardiovasc Dis. 45: 173-202.

23. Julian CG, Vargas E, Armaza JF, Wilson MJ, Niermeyer S, Moore LG. 2007. High-altitude ancestry protects against hypoxia-associated reductions in fetal growth. Arch Dis Child Fetal Neonatal Ed. 92(5):F372-7.

24. Keyes LE, Armaza JF, Niermeyer S, Vargas E, Young DA, Moore LG. 2003. Intrauterine growth restriction, preeclampsia, and intrauterine mortality at high altitude in Bolivia. Pediatr Res 54: 20-25.

25. León-Velarde F, Richalet JP. 2006. Respiratory control in residents at high altitude: physiology and pathophysiology. High Alt Med Biol. 7(2):125-37.

26. Monge C, Leon-Velarde F. 1991. Physiological adaptation to high altitude: oxygen transport in mammals and birds. Physiol Rev. 71: 1135-72.

27. Nachar RA, Pastene CM, Herrera EA, Riquelme RA, Sanhueza EM, Troncoso S, Llanos AJ. 2001. Low-dos inhaled intrauterine growth: comparison between high- and low-altitude native ewes. Reprod Fertil Dev. 17(5):497-505. Carbon monoxide reduces pulmonary vascular resistance during acute hypoxemia in adult sheep. High Alt Med Biol. 2: 377-385.

28. Nash ML, Hungerford LL, Nash TG, Zinn GM. 1996. Risk factors for perinatal and postnatal mortality in lambs. Vet Rec. 139(3): 64-7.

29. Ou LC, Chen J, Fiore E, Leiter JC, Brinck-Johnsen T, Birchard GF, Clemons G, Smith RP. 1992. Ventilatory and hematopoietic responses to chronic hypoxia in two rat strains. J Appl Physiol. 72(6):2354-63.

30. Parraguez VC, Atlagich M, Díaz R, Bruzzone ME, Behn C, Raggi LA. 2005. Effect of hypobaric hypoxia on lamb

31. Resta TC, Broughton BR, Jernigan NL. 2010. Reactive oxygen species and RhoA signaling in vascular smooth muscle: role in chronic hypoxia-induced pulmonary hypertension. Adv Exp Med Biol. 661:355-73.

32. Rhodes J. 2005. Comparative physiology of hypoxic pulmonary hypertension: historical clues from brisket disease. J Appl Physiol. 98(3):1092-100. 
33. Spieker LE, Flammer AJ, Lüscher TF. 2006. The vascular endothelium in hypertension. Handb Exp Pharmacol. 176 (Pt 2):249-83.

34. Stenmark KR, Fagan KA, Frid MG. 2006. Hypoxia-induced pulmonary vascular remodeling: cellular and molecular mechanisms. Circ Res. 99(7):675-91.

35. Tucker A, Mc Murtry IF, Reeves JT, Alexander AF, Will DH, Grover RF. 1975. Lung vascular smooth muscle as a determinant of pulmonary hypertension at altitude. Am. J. Physiol. 228: 762767.

36. Weir EK, Cabrera JA, Mahapatra S, Peterson DA, Hong Z. 2010. The role of ion channels in hypoxic pulmonary vasoconstriction. Adv Exp Med Bio. 